\title{
Identification of Main Phenolic Compounds of Nitraria Retusa Leaf and Stem Extracts and Evaluation of Their Anti- Adipogenic Activity in 3T3-L1 Cells
}

Feten Zar Kalai ( $\sim$ zarfeten@gmail.com )

Centre de Biotechnologie de Borj Cedria

Mondher Boulaaba

Centre de Biotechnologie de Borj-Cédria https://orcid.org/0000-0002-3488-5316

Riadh Ksouri

Centre de Biotechnologie de Borj Cedria

\section{Hiroko Isoda}

University of Tsukuba: Tsukuba Daigaku

\section{Research Article}

Keywords: Nitraria retusa, Phenolic compounds, adipogenesis, Phytochemical

Posted Date: September 3rd, 2021

DOI: https://doi.org/10.21203/rs.3.rs-868059/v1

License: (c) (i) This work is licensed under a Creative Commons Attribution 4.0 International License. Read Full License 


\section{Abstract}

The edible halophyte Nitraria retusa known in traditional medicine purposes was used as a support in this study. The present study investigated the chemical analyses of the general composition of leaf and stem extracts using RP-HPLC. Results showed the richness of these extracts in phenolics especially flavonoids as luteolin-7-0-glucoside, isorhamnetin-3O-rutinoside, isorhamnetin, quercetin and others. In total, nine compounds were identified for each organ extract. Luteolin-7O-glucoside, isorhamnetin-3-0-rutinoside and Isorhamnetin are three flavonoids which are common in leaves and stems. Adipogenesis assay was performed to investigate the effect of organs, as well as the three last compounds on the adipocyte differentiation and on the lipid droplets accumulation in 3T3-L1 cells. Based on Oil-Red-O content quantification in 3T3-L1 cells, results showed that each extract of $N$. retusa at 25, 50, 100, 200 and $400 \mu \mathrm{g} / \mathrm{mL}$ could not inhibit the lipid droplet accumulation compared to untreated cells, in dose dependent manner. However, compounds showed better effect especially with isorhamnetin even with $5 \mu \mathrm{M}$. Moreover, the effect of extracts and single bioactive components on cell proliferation of 3T3-L1 cells showed that stem extract was more efficient than leaf one. Besides, according to morphological observation, isorhamnetin strongly and significantly affected cell proliferation.

\section{Introduction}

The plant kingdom has become a target for the search by multinational drug companies and research institutes for new drugs and biologically active compounds [1]. The world health organization (WHO) reported in 2002 that the herbal medicine serves the health need of about $80 \%$ of the world's population, especially for millions of people in the vast rural areas of developing countries [2]. As known actually, phenolic compounds are receiving increasing attention because of their health promoting effects, attributed to their various biological activities and strong capacity to improve food quality and stability [3]. Phenolic compounds represent the most studied phytochemicals and have been widely exploited as model systems in different areas of plant research such as pharmaceutical, food, cosmetic and chemical industries [4]. Improving knowledge on the use of anti-obesity medicinal preparations, and encouraging obese patients to consume them along with a healthy diet should be continued [5]. In order to use safely the medicinal plants in controlling and treating obesity, further chemical, biological, and clinical studies are needed on the efficiency of selected plants. Plant extracts may be the whole plant or plant organs (stems, leaves, flowers, and roots). Several investigations should focus on the difference between the combination and the single effect of such compounds. In view of the diversity and the structural complexity of these bioactive molecules, their extraction is influenced by several parameters and technology including the nature, polarity, extracting power of the solvent and extraction method [6; 7]. Other factors influence the extraction and activities of compounds are genotype of plants as well as their physiological state, organs and environmental conditions such as temperature, salinity, drought, light intensity and bacterial infection $[8 ; 9 ; 10 ; 11 ; 12]$.

High performance liquid chromatography (HPLC) is the method of choice for the analysis of phenolic compounds, because of its versatility, precision and relatively low cost [13]. Most frequently, reversed-phase (RP) C18 columns, a binary solvent system containing acidified water and polar organic solvent (acetonitrile or methanol), and UV-Vis diode array detection (DAD) are used and so far to constitute a crucial and reliable tool in the routine analysis of plant phenolic compounds [14].

In this present work we propose to study Nitraria retusa plant, a North African halophyte of the Nitrariaceae family (formerly belonging to the Zygophyllaceae) well adapted to the arid climate thanks in particular to its fleshy leaves and also known in Tunisia under the name of "Ghardak". N. retusa is known for their biological effects. A previous study was carried out on this plant by some authors of this present work in order to enhance the role in the control of cellular accumulation of fat [15]. In addition, research in Morocco has shown that fresh leaves of $N$. retusa serve as a remedy for poisoning and stomach and abdominal pain [16]. N. retusa is also known for its antioxidant, antiproliferative [17] and antimicrobial [18] activities. In addition, the fruits of $N$. retusa are also used as a treatment for hypertension and difficult menstruation [17]. On the other hand, older studies have shown that $N$. retusa is also known for its effect against swelling, its ability to remove tumors and as a traditional remedy for infected wounds [19]. Regarding the phytochemical profile, the genus Nitraria has 
long been known for its richness in polyphenols, mainly the group of flavonoids such as isorhamnetin with strong biological activities, particularly antioxidant and antiproliferative [20; 17].

The aim of this work is to analyze the phenolic composition of Nitraria retusa extracts (stem and leaf extracts). In a further step, we aim to test the anti-adipogenic effect of extracts on 3T3-L1 cells as well as some of their identified phenolic compounds. At the end and to better understand the treatment effects on cells, the morphological observations as well as their proliferative analysis were done.

\section{Materials And Methods}

\subsection{Plant sampling and extracts preparation}

Plant samples of different parts of Nitraria retusa were collected from a salt flat "sebkha of El Kelbia" located in the region of Kairouan, Tunisia with the GPS following coordinates N 3548 44, E 1009 06. After rinsing samples with distilled water and dried in shadow. The organs of $N$. retusa (stems and leaves) were ground separately using a ball mill type "Dangoumeau" and extracted with 70\% of ethanol with a ratio of $1 \mathrm{~g}$ of plant powder in $10 \mathrm{ml}$ of extraction solvent. Then the solution was kept in the dark for 2 weeks. During this period, the extract solution was manually shaked for 5-10 minutes every day. Finally, the extract was filtered through $0.22 \mu \mathrm{m}$ filter (MILLIPORE, U.S.A.), and stored for further experiments [21].

\subsection{Chemical analyses of the general composition of Nitraria retusa extracts (stem extract and leaf extract) using reverse phase-high performance liquid chromatography (RP-HPLC)}

Before injection into the HPLC system, $N$. retusa organ extracts were passed through a $0.45 \mu \mathrm{m}$ nylon filter. The separation of selected phenolic compounds was carried out using HPLC system (consisting of a vacuum degasser, an autosampler, and a binary pump with a maximum pressure of 600 bar, Agilent 1260, Agilent technologies, Germany) equipped with a reversed phase $\mathrm{C} 18$ analytical column of $4.6 \times 100 \mathrm{~mm}$ and $3.5 \mu \mathrm{m}$ particle size (Zorbax Eclipse XDB C18). Column temperature was maintained at $25^{\circ} \mathrm{C}$. The injected sample volume was $2 \mu \mathrm{L}$ and the flow-rate of mobile phase was 0.4 $\mathrm{mL} / \mathrm{min}$. Mobile phase $B$ was milli-Q water consisted of $0.1 \%$ formic acid and mobile phase $A$ was methanol. The optimized chromatographic condition was revealed as follows: $10 \% \mathrm{~A}, 90 \% \mathrm{~B}$ (0 min), 20\% A, 80\% B (5 min), 30\% A, 70\% B (10 min), 50\% A, 50\% B (15 min), 70\% A, 30\% B (20 min), 90\% A, 10\% B (25 min), 50\% A, 50\% B (30 min), 10\% A,90\% B (35 $\mathrm{min})$. UV-vis absorption spectra were recorded online during the HPLC analysis. The DAD detector was set to a scanning range of 200-400 nm. Peak identification was obtained comparing the retention time and the UV-vis spectra of Nitraria retusa phenolics chromatogram with those of available standards [22]. Quantification was performed by reporting the measured integration area in the calibration equation of the corresponding standard.

\subsection{Preadipocytes differentiation and Oil-Red-O staining procedures}

3T3-L1 preadipocytes were seeded into 96-well plates at $1.0 \times 104$ cells/well and cultured for additional two days until full confluence. Two days later (Day 0), cells were incubated with a differentiation cocktail (MDI) containing 1/10 insulin solution, 1/10 dexamethasone solution and 1/10 3-isobutyl-1-methylxanthine solution in standard culture medium for 3 days followed by additional $48 \mathrm{~h}$ with standard culture medium containing insulin alone. The differentiation-maintenance medium was changed every 2 days [21]. To investigate the effect $N$. retusa stem and leaf extracts on adipogenesis in 3T3$\mathrm{L} 1$, different doses $(25,50,100,200$ and $400 \mu \mathrm{g} / \mathrm{mL})$ were added to the differentiation-induction and differentiationmaintenance media. The same procedure was conducted to investigate the effect of the determined phenolic compounds of $N$. retusa (luteolin-7-0-glucoside, isorhamnetin-3-O-rutinoside and isorhamnetin) using the following concentrations: 5 , $25,50,100$ and $200 \mu \mathrm{M}$. The staining procedure was conducted according to the adipogenesis assay kit (Cayman chemical company). The absorbance was read at $490 \mathrm{~nm}$ with a 96-well plate reader. The lipid droplet content was reported as percentage of control cells. 


\subsection{Cell culture}

Murine 3T3-L1 preadipocytes (Riken Tsukuba Japan) were maintained in Dulbecco's modified Eagle's medium (DMEM) supplemented with $10 \%$ fetal bovine serum (FBS) and $1 \%$ penicillin $(5000 \mu \mathrm{g} / \mathrm{mL})$-streptomycin $(5000 \mathrm{IU} / \mathrm{mL})$ in $75-\mathrm{cm} 2$ tissue culture flasks. Medium was changed every 3 days and cell passage was carried out at $80 \%$ confluence at one on two ratio using $0.25 \%$ trypsin ( $1 \mathrm{mM}$ EDTA). 3T3-L1 cells were cultured in a humidified incubator at $37^{\circ} \mathrm{C}$ and $5 \% \mathrm{CO} 2$ [21].

\subsection{Cell proliferation assay (MTT assay)}

Cell proliferation was investigated by MTT (3-(4,5-Dimethylthiazol-2-yl)-2,5-diphenyltetrazolium bromide) assay. 3T3-L1 cells were seeded in 96-well plates at $1 \times 105$ cells $/ \mathrm{mL}$. After incubation for 7 days (adipocytes), leaf and stem extract samples diluted in medium were added at final concentrations of $25,50,100,200,400 \mu \mathrm{g} / \mathrm{mL}$. The same procedure was conducted to investigate the effect of the determined phenolic compounds of $N$. retusa using 5, 25, 50, 100 and $200 \mu \mathrm{M}$ concentrations for luteolin-7-0-glucoside, isorhamnetin-3-0-rutinoside and isorhamnetin. MTT was added after treatment for 7 days and the resulting formazan was completely dissolved by $100 \mu \mathrm{L}$ of $10 \%$ sodium dodecyl sulfate (SDS) for $24 \mathrm{~h}$. The absorbance was determined at $570 \mathrm{~nm}$ in a multi-detection microplate reader (Power-scan HT, Dainippon Pharmaceutical, NJ, USA). Absorbance caused by the ability of the sample to reduce MTT or by its color, was corrected using plates as blanks, prepared in the same conditions in the absence of cells.

\section{Results}

\subsection{Chemical analyses of the general composition of Nitraria retusa extracts using reverse phase-high performance liquid chromatography (RP-HPLC)}

After analysis, nine compounds were identified for each organ extract (Table 1). Peak identifications were obtained comparing the retention time and the UV spectra of Nitraria retusa phenolics chromatogram with those of available standards (Table 2). The chromatographic profiles of $N$. retusa extracts (stems and leaves), acquired at $254 \mathrm{~nm}$, were presented in the figures 1 and 2 respectively. Quantification was performed by reporting the measured integration area in the calibration equation of the corresponding standard. Luteolin-7-O-glucoside, isorhamnetin-3-O-rutinoside and Isorhamnetin are three flavonoids which are common in leaves and stems. According to quantification measurements, the concentrations in leaf extract $(\mu \mathrm{g} / \mathrm{mL})$ of isorhamnetin, isorhamnetin-3-O-rutinoside and luteolin-7-O-glucoside were 0.539, 278.01 , and 252.403, respectively (Table 1$)$. In other side, the concentrations in stem extract $(\mu \mathrm{g} / \mathrm{mL})$ of isorhamnetin, isorhamnetin-3-O-rutinoside and luteolin-7-O-glucoside were 1.463, 130.417, and 7.902, respectively (Table 1). These flavonoids were chosen for further experiments to test the single effects of $N$. retusa components on cell viability, differentiation and lipid droplet formation in 3T3-L1 cells.

\subsection{Effect of Nitraria retusa crude extracts and single bioactive components on cell differentiation and lipid droplet formation in 3T3-L1 cells}

Adipogenesis assay was performed to investigate the effect of Nitraria retusa extracts (stem and leaf extracts), as well as the effect of selected bioactive components (Luteolin-7-O-glucoside, isorhamnetin-3-O-rutinoside and isorhamnetin) on the adipocyte differentiation and on the lipid droplets accumulation in 3T3-L1 cells. Differentiated 3T3-L1 cells were treated every two days with the different extracts described above and at various concentrations $(25,50,100,200 \mathrm{and} 400 \mu \mathrm{g} / \mathrm{mL})$ for 7 days. Moreover, in order to compare the effect of single compounds to the extract one, differentiated 3T3-L1 cells were treated every two days with various concentrations of selected chemical compounds $(5,25,50,100$ and $200 \mu \mathrm{M})$. Based on Oil-Red-O content quantification in cells, results showed that each extract of $N$. retusa at 25, 50, 100, 200 and 400 $\mu \mathrm{g} / \mathrm{mL}$ could not inhibit the lipid droplet accumulation compared to untreated cells, in dose dependent manner (Fig 3). Regarding the effect of compounds identified in $N$. retusa extracts on adipogenesis, results showed that dependent on the chemical structure and molecular weights of all compounds tested, there is a variability of their effect on 3T3-L1 
differentiation and triglyceride accumulation within cells (Fig 4). Actually, isorhamnetin showed the strongest effect for all tested doses even for the lowest one $(5 \mu \mathrm{M})$, compared to the other flavonoids glycosides (isorhamnetin-3-O-rutinoside and luteolin-7-O-glucoside). In fact, after treating cells with isorhamnetin in various doses $(5,25,50,100,200 \mu \mathrm{M})$, the triglyceride accumulation significantly decreased to $24.45 \%, 24.45 \%, 28.33 \%, 31.31 \%$, and $20.57 \%$, respectively (Fig $4 \mathrm{C}$ ). However, high doses of luteolin-7-O-glucoside and isorhamnetin-3-O-rutinoside (50, 100, $200 \mu \mathrm{M})$ exhibited a significant reduction of lipid accumulation (Fig 4A and B), accompanied with cell morphological changes based on microscopic observation (Fig 4D). Therefore, it is necessary to correlate the obtained results with the MTT assay results in next part.

\subsection{Effect of Nitraria retusa extracts and single bioactive components on cell proliferation of 3T3-L1 cells}

The effects of leaf and stem extracts and compound ones (Luteolin-7-0-glucoside, isorhamnetin-3-0-rutinoside and isorhamnetin) on the morphology and the proliferation of 3T3-L1 adipocytes cells are presented in the figures 5 and 6. Results showed that treatments with the two organs of $N$. retusa revealed no significant decrease in cell proliferation as well as no changes in the morphology with the lower concentrations ( 25 and $50 \mu \mathrm{g} / \mathrm{mL}$ ). However, at the concentrations of 100, 200 and $400 \mu \mathrm{g} / \mathrm{mL}$, stem extract significantly inhibited the growth of 3T3-L1 cells as compared to the control one by $20 \%$ for the two first doses and $30 \%$ for $400 \mu \mathrm{g} / \mathrm{mL}$ (Fig 5B). Concerning the tested single compounds, cell proliferation was slightly significant affected by isorhamnetin-3-O-rutinoside treatments $(25,50,100,200 \mu \mathrm{M})$ with just $5 \%$ maximum of cell growth reduction for the higher dose (Fig 6B). Luteolin-7-O-glucoside treatments affected more significantly the cell proliferation by decreasing it by a maximum around 40\% using $100 \mu \mathrm{M})(\mathrm{Fig} 6 \mathrm{~A})$. Nonetheless, isorhamnetin strongly affected the 3T3-L1 growth by $30 \%$ and $60 \%$ reduction for 100 and $200 \mu \mathrm{M}$, respectively (Fig $6 \mathrm{C}$ ).

\section{Discussion}

The main idea of this study was to give an explanation to the anti-adipogenic activity of Nitraria retusa extracts of stems and leaves by the analysis of the phytochemical using the RP-HPLC system. Observation of results revealed the presence of many phenolics especially flavonoids as luteolin-7-0-glucoside, isorhamnetin-3-0-rutinoside, isorhamnetin, quercetin and others. In total, nine compounds were identified for each organ. Luteolin-7-0-glucoside, isorhamnetin-3-0-rutinoside and isorhamnetin are three flavonoids which are common in leaves and stems. For this work, $70 \%$ of ethanol was used for the extraction. It's important to mention that on the one hand, extraction is influenced by several parameters and technology including the nature, polarity, extracting power of the solvent and extraction method [6; 7]. On the other hand, genotype of plants as well as their physiological state, organs and environmental conditions such as temperature, salinity, drought, light intensity and bacterial infection influence extraction and activities of extracts and compounds [8; 9; 10; 11; 12]. Moreover, some of these compounds were already detected in other halophytes as the case of quercetin, isorhamnetin-3-0-rutinoside and isorhamnetin (176.33, 157 and $86.76 \mu \mathrm{g} / \mathrm{g}$ DW, respectively) in Limoniastrum guyonianum roots [23]. Isorhamnetin glycosides were also isolated from Peucedanum aucheri [24] and Arthrocnemum glaucum [25]. Resorcinol, hyperoside and quercetin were also detected in Salsola kali with different amounts in the leaves and/or stems respectively expressed as milligram per gram of residue (R): (0.09295 and 0.21138; 0.14912 and 0.18838 ; and $\left.0.05178 \mathrm{mg} \mathrm{g}^{-1} \mathrm{R}\right)$ [12].

All molecules in plants could work in synergy and structure of each compound could be the origin of the intensity of the biological effects $[14 ; 20 ; 17 ; 26]$. In fact, the anti-adipogenic activity of $N$. retusa may be the result of its richness in phenolics, more probably in flavonoids as isorhamnetin and their combined activity or synergism. The lower antiadipogenic activity of organ extracts compared to their single constituents could be explained by the concentrations of some compounds present in $N$. retusa [27]. In our study, isorhamnetin was more effective compared to the other compounds (isorhamnetin-3-O-rutinoside, luteolin-7-O-glucoside) even in the lowest dose $(5 \mu \mathrm{M})$. For more understanding, the anti-adipogenic effect was previously showed with isorhamnetin in 3T3-L1 cells by reducing adipogenic gene expression as C/EBP-a and PPAR-y [28] and the activation of the Wnt/ $\beta$-catenin signaling [29]. Same tendency was observed with luteolin-7-0-glucoside from Commelina communis, an Asian dayflower, and the results showed that this molecule control lipogenesis by the reducing of the transcription level of glucose transporter 4 (GLUT4) and glucose uptake 
[30]. Moreover, the strong activity of isorhamnetin, as an 0-methylated flavonol of quercetin, could be due to the contribution of its chemical structure since it is the only compound of $N$. retusa extracts possessing a methoxy group at a 3-position and glucose free. In this context, it was reported that flavonoids with methoxy groups showed stronger antidiabetic effects particularly those with a methoxy group at the 3-position [31]. It has been also reported that several flavonoids with methoxyl substitutions exhibited higher anti-cancer activities which explain the antiproliferative effect of isorhamnetin compound [32]. As well, it was reported in comparison study between isorhamnetin free and isorhamnetin glycosides on their anti-oxidant activities in vivo system that isorhamnetin glycosides is metabolized in vivo by intestinal bacteria to isorhamnetin which plays important biological roles as among them antioxidant effect [33]. In fact, previous studies confirmed that the biological activities depend on the presence or absence of the glycoside residue [17; 34]. However, in addition to the efficiency parameter, safety of tested doses of different compounds should be considered. Isorhamnetin in single effect showed a reduction on cell proliferation of 3T3-L1 cells compared to other glycosides compounds (Fig. 6).

As mentioned previously in this discussion, the composition of crude extract will depend on the solvent used and also on the quality of the original material, and its composition. In order to obtain a high quality extract with antioxidant activity that is suitable for use in the food, cosmetic, and pharmaceutical industries, the extract must be purified to remove all inert and undesirable components. A purification and fractionation processes that remove fractions with limited antioxidant activity might enable a good level of biological activity. Then, fractionation, purification, and/or the isolation of active principle(s) could increase bioactive component bioavailability in the extracts, and improve medicinal agent efficacy in weight loss [35; 36].

Because of their benefits to human health, natural antioxidants isolated from $N$. retusa could be an alternative used in food industries in order to avoid or at least reduce the use of synthetic products. Further chemical, biological, and clinical studies are needed on the effectiveness of selected medicinal plants in ameliorating and treating obesity in humans, in order to improve our knowledge on the use of anti-obesity medicinal herbal preparations.

\section{Conclusion}

The extracts of stems and leaves from Nitraria retusa tested in the current study were in crude form but contained many bioactive components which may well act in synergy. To determine which compounds are responsible for the observed effects further experiments of bioactivity-guided fractionation and isolation of bioactive compounds are required.

\section{Declarations}

\section{Conflict of Interests}

The authors declare that they have no conflict of interests.

\section{Acknowledgment}

This research was supported by the JICA/JST Science and Technology Research Partnership for sustainable Development (SATREPS) Project: "Valorization of Bio-resources in SemiArid and Arid Land for Regional Development."

\section{References}

1. Jesse WH Li, John C Vederas. Drug Discovery and Natural Products: End of an Era or an Endless Frontier? Science. 2009,161-165. doi: 10.1126/science.1168243.

2. Ekor M. The growing use of herbal medicines: issues relating to adverse reactions and challenges in monitoring safety. Front. Pharmacol. 2014,4:177. doi: 10.3389/fphar.2013.00177. 
3. Meccariello R, D'Angelo S. Impact of Polyphenolic-Food on Longevity: An Elixir of Life. An Overview. Antioxidants 2021,10:507. Doi: 10.3390/antiox10040507.

4. Boudet A.M. Evolution and current status of research in phenolic compounds. J. Phytochem. 2007,68:2722-2735. https://doi.org/10.1016/j.phytochem.2007.06.012.

5. Kazemipoor M, Radzi CWJWM, Cordell GA, Yaze I. Potential of traditional medicinal plants for treating obesity: a review. IPCBEE, Singapore. 2012,39(1):1-6.

6. Kaczorová D, Karalija E, Dahija S, Bešta-Gajević R, Parić A, Ćavar Zeljković S. Influence of Extraction Solvent on the Phenolic Profile and Bioactivity of Two Achillea Species. Molecules. 2021,26(6):1601. doi:10.3390/molecules26061601.

7. Trabelsi N, Megdiche W, Ksouri R, Falleh H, Oueslati S, Bourgou S, Hajlaoui H, Abdelly C. Solvent effects on phenolic contents and biological activities of the halophyte Limoniastrum monopetalum leaves. LWT-Food Sci Technol. 2010,43:632-639. doi: 10.1016/j.Iwt.2009.11.003.

8. Lisiewska Z, Kmiecik,W, Korus. Content of vitamin C, carotenoids, chlorophylls and polyphenols in green parts of dill (Anethum graveolens L.) depending on plant height. J Food Compost Anal. 2006,19:134-140. doi:10.1016/j.jfca.2005.04.009.

9. Karray-Bouraoui N, Ksouri R, Falleh H, Rabhi M, Abdul Jaleel C, Grignon C. Lachaâl M. Effect of environment and development stage on phenolic content and antioxidant activities of Mentha pulegium L. J Food Biochem. 2010,34:79-89. Doi.org/10.1111/j.1745-4514.2009.00303.x.

10. Medini F, Ksouri R, Falleh $\mathrm{H}$ et al.. Effects of physiological stage and solvent on polyphenol composition, antioxidant and antimicrobial activities of Limonium densiflorum. J Med Plants Res 2011,5:6719-6730. doi: 10.5897/JMPR11.684.

11. Jallali I, Megdiche W, M'Hamdi B, Oueslati S, Smaoui A, Abdelly C, Ksouri R. Changes in phenolic composition and antioxidant activities of the edible halophyte Crithmum maritimum L. with physiological stage and extraction method. Acta Physiol Plant. 2012, 34:1451-1459. doi: 10.1007/s11738-012-0943-9.

12. Boulaaba M, Zar Kalai F, Dakhlaoui S, et al. Antioxidant, antiproliferative and anti-inflammatory effects of Glaucium flavum fractions enriched in phenolic compounds. Med Chem Res 2019, 28:1995-2001.

13. Tsao R, Yang R. Optimization of a new mobile phase to know the complex and real polyphenolic composition: towards a total phenolic index using high-performance liquid chromatography. J Chromatogr A. 2003,1018(1):29-40. doi: 10.1016/j.chroma.2003.08.034.

14. Naczka M, Shahidi F. Extraction and analysis of phenolics in food. J Chromatogr A. 2004,1054(1-2):95-111. doi: 10.1016/j.chroma.2004.08.059.

15. Zar Kalai F, Han J, Ksouri R, et al. Oral administration of Nitraria retusa ethanolic extract enhances hepatic lipid metabolism in db/db mice model 'BKS.Cg-Dock7+/+ Leprdb/J' through the modulation of lipogenesis-lipolysis balance. Food Chem Toxicol 2014,72:247-256. doi: 10.1016/j.fct.2014.07.029.

16. Boubaker J, Skandrani I, Bouhlel I, Ben Sghaier M, Neffati A, Ghedira K, Chekir-Ghedira L. Mutagenic, antimutagenic and antioxidant potency of leaf extracts from Nitraria retusa. Food Chem Toxicol. 2010,48(8-9):2283-90. doi: 10.1016/j.fct.2010.05.061.

17. Hadj Salem J, Chevalot I, Harscoat-Schiavo C, et al. Biological activities of flavonoids from Nitraria retusa (Forssk.) Asch. and their acylated derivatives. Food Chem. 2011, 124:486-494. doi: 10.1016/j.foodchem.2010.06.059.

18. Chaâbane M, Soudani N, Benjeddou K, Turki K, Ayadi Makni F, Boudawara T et al. The protective potential of Nitraria retusa on penconazole-induced hepatic injury in adult rats. Toxicol Environ Chem. 2015,97(9):1253-1264. doi: 10.1080/02772248.2015.1093633

19. Chaâbane M, Koubaa M, Soudani N, Elwej A, Grati M, Jamoussi K et al. Nitraria retusa fruit prevents penconazoleinduced kidney injury in adult rats through modulation of oxidative stress and histopathological changes. Pharm Biol. 
2017,55(1):1061-1073. doi: 10.1080/13880209.2016.1278455.

20. Hussein SR, Kawashty SA, Tantawy ME, Saleh NAM. Chemosystematic studies of Nitraria retusa and selected taxa of Zygophyllaceae in Egypt. Plant Syst Evol. 2009,277:251-264. doi: 10.1007/s00606-008-0108-x.

21. Zar Kalai F, Boulaaba M, Trabelsi N, Waffo Teguo P, Mérillon Jean M, Smaoui A, Ksouri R. Phenolic content and biological activities of Limonium densiflorum crude extract and its methanolic fraction: Influence of genotype and fractionation. Int J Med Plant. Photon 2013,105:334-349.

22. Guyot S, Marnet N, Laraba D, Sanoner P, Drilleau JF. Reversed-phase HPLC following thiolysis for quantitative estimation and characterization of the four main classes of phenolic compounds in different tissue zones of a rench cider apple variety (Malus domestica Var. Kermerrien). J Agric Food Chem. 1998,46:1698-1705. doi: 10.1021/jf970832p.

23. Bettaib J, Talarmin H, Kalai FZ, Giroux-Metges MA, Ksouri R. Limoniastrum guyonianum prevents H2O2-induced oxidative damage in IEC-6 cells by enhancing enzyamtic defense, reducing glutathione depletion and JNK phosphorylation, Biomed Pharmacother. 2017. 95:1404-1411. doi: 10.1016/j.biopha.2017.09.068.

24. Dehaghani ZA, Asghari G, Dinani MS. Isolation and Identification of Nicotiflorin and Narcissin from the Aerial Parts of Peucedanum aucheri Boiss. J Agric Sci Technol A. 2.017,7:45-51. doi:10.17265/2161-6256/2F2017.01.007.

25. Sekii Y, Han J, Isoda H. et al. Two isorhamnetin glycosides from Arthrocnemum glaucum that inhibit adipogenesis in 3t3-I1 adipocytes. Chem Nat Compd. 2015,51, 338-340. doi.org/10.1007/s10600-015-1276-x.

26. Boubaker J, Sghaier MB, Ines S, Ghedira K, Chekir-Ghedira L. Isorhamnetin 3-0-robinobioside from Nitraria retusa leaves enhance antioxidant and antigenotoxic activity in human chronic myelogenous leukemia cell line K562. BMC Complement Altern Med. 2012,12:135. doi.org/10.1186/1472-6882-12-135.

27. Lee J, Jung E, Lee J, Kim S, Huh S, Kim Y, Kim Y, Byun SY, Kim YS, Park D. Isorhamnetin Represses Adipogenesis in 3T3-L1 Cells. Obesity. 2009,17(2):226-232. doi:10.1038/oby.2008.472.

28. Lee SH, Kim B, Oh MJ, Yoon J, Kim HY, Lee KJ, Lee JD, Choi KY. Persicaria hydropiper (L.) Spach and its Flavonoid Components, Isoquercitrin and Isorhamnetin, Activate the Wnt/ $\beta$-catenin Pathway and Inhibit Adipocyte Differentiation of 3T3-L1 Cells. Phytother Res. 2011,25:1629-1635.doi: 10.1002/ptr.3469.

29. Nagai S, Wakai E, Shibano M, Fujimori K. Anti-obesity effects of Asian dayflower, Commelina communis, in mice with high-fat diet-induced obesity and in 3T3-L1 cells. J Funct Foods 2016,22:490-503. doi: 10.1016/j.jff.2016.02.012.

30. Matsuda H, Kogami Y, Nakamura S, Sugiyama T, Ueno T, Yoshikawa, M. Structural requirements of flavonoids for the adipogenesis of 3T3-L1 cells. Bioorg Med Chem. 2011,19(9), 2835-2841. doi:10.1016/j.bmc.2011.03.040.

31. Boubaker J, Bhouri W, Ben Sghaier M, Ghedira K, Dijoux-Franca MG, Chekir-Ghedira L. Ethyl Acetate Extract and Its Major Constituent, Isorhamnetin 3-0-Rutinoside, from Nitraria retusa Leaves, Promote Apoptosis of Human Myelogenous Erythroleukaemia Cells. Cell Prolif. 2011,44(5):453-61.

32. Yokozawa T, Kim HY, Cho EJ, Choi JS, Chung HY. Antioxidant effects of isorhamnetin 3,7-Di-O- $\beta$-d-glucopyranoside isolated from mustard leaf (Brassica juncea) in rats with streptozotocin-induced diabetes. J Agric Food Chem. 2002,50:5490-5495. doi:10.1021/jf0202133.

33. Xiao J. Dietary flavonoid aglycones and their glycosides: Which show better biological significance? Crit Rev Food Sci Nutr. 2017,57(9):1874-1905.. doi: 10.1080/10408398.2015.1032400.

34. Sheng X, Zhang Y, Gong Z, Huang C, Zang YQ. Improved insulin resistance and lipid metabolism by cinnamon extract through activation of peroxisome proliferator-activated receptors. PPAR Res. 2008,1-9. doi: 10.1155/2008/581348.

35. Calixto JB. 2003. Efficacy, safety, quality control, marketing and regulatory guidelines for herbal medicines (phytotherapeutic agents). Braz J Med Biol Res. 2003,33:179-189. doi: 10.1590/s0100-879x2000000200004.

\section{Tables}


Table 1. Identification and quantification of phenolic compounds of leaf and stem extracts of Nitraria retusa

\begin{tabular}{|c|c|c|c|c|c|c|c|c|c|c|}
\hline \multirow[t]{2}{*}{ Identifications } & \multicolumn{2}{|l|}{ Peaks } & \multicolumn{2}{|c|}{$\begin{array}{l}\text { Retention times } \\
(\min )\end{array}$} & \multicolumn{2}{|l|}{ Area } & \multicolumn{2}{|c|}{$\begin{array}{l}\text { Quantifications } \\
(\mu \mathrm{g} / \mathrm{mL})\end{array}$} & \multicolumn{2}{|c|}{$\begin{array}{l}\text { Quantifications } \\
\text { (mg/g extract) }\end{array}$} \\
\hline & Leaves & Stems & Leaves & Stems & Leaves & Stems & Leaves & Stems & Leaves & Stems \\
\hline Resorcinol & 1 & 1 & 10.813 & 10.882 & 27.2 & 30.5 & 69.152 & 77.542 & 2.305 & 2.584 \\
\hline $\begin{array}{l}\text { Chlorogenic } \\
\text { acid }\end{array}$ & 2 & 2 & 15.685 & 15.882 & 150 & 43.2 & 20.690 & 25.958 & 0.689 & 0.865 \\
\hline $\begin{array}{l}P \text {-coumaric } \\
\text { acid }\end{array}$ & - & 3 & - & 20.010 & - & 13.7 & - & 3.335 & - & 0.111 \\
\hline Sinapic acid & 3 & 4 & 20.220 & 20.306 & 66.6 & 30.1 & 9.651 & 4.361 & 0.321 & 0.145 \\
\hline $\begin{array}{l}\text { Luteolin 7-O- } \\
\text { glucoside }\end{array}$ & 4 & 5 & 21.191 & 21.179 & 2539.3 & 79.5 & 252.403 & 7.902 & 8.413 & 0.263 \\
\hline Isoquercitrin & 5 & - & 21.756 & - & 23.5 & - & 16.708 & - & 0.556 & - \\
\hline Hyperoside & 6 & 6 & 21.866 & 21.875 & 158.5 & 63 & 6.388 & 2.539 & 0.212 & 0.084 \\
\hline $\begin{array}{l}\text { Isorhamnetin } \\
3-0 \text { - } \\
\text { rutinoside }\end{array}$ & 7 & 7 & 22.839 & 22.804 & 1523.1 & 714.5 & 278.010 & 130.417 & 9.269 & 4.347 \\
\hline Quercetin & 8 & 8 & 24.473 & 24.559 & 23.9 & 23.6 & 1.828 & 1.805 & 0.060 & 0.060 \\
\hline Isorhamnetin & 9 & 9 & 25.840 & 25.840 & 11.9 & 32.3 & 0.539 & 1.463 & 0.017 & 0.048 \\
\hline
\end{tabular}

Table 2. List of standards and their retention times (min).

\begin{tabular}{|ll|}
\hline Standards & Retention time (min) \\
\hline Resorcinol & 10.835 \\
\hline Chlorogenic acid & 15.918 \\
\hline P-coumaric acid & 20.091 \\
\hline Sinapic acid & 20.278 \\
\hline Luteolin-7-O-glucoside & 21.145 \\
\hline Isoquercetin & 21.745 \\
\hline Hyperoside & 21.803 \\
\hline Isorhamnetin-3-O-rutinoside & 22.986 \\
\hline Quercetin & 24.285 \\
\hline Isorhamnetin & 25.899 \\
\hline
\end{tabular}

\section{Figures}




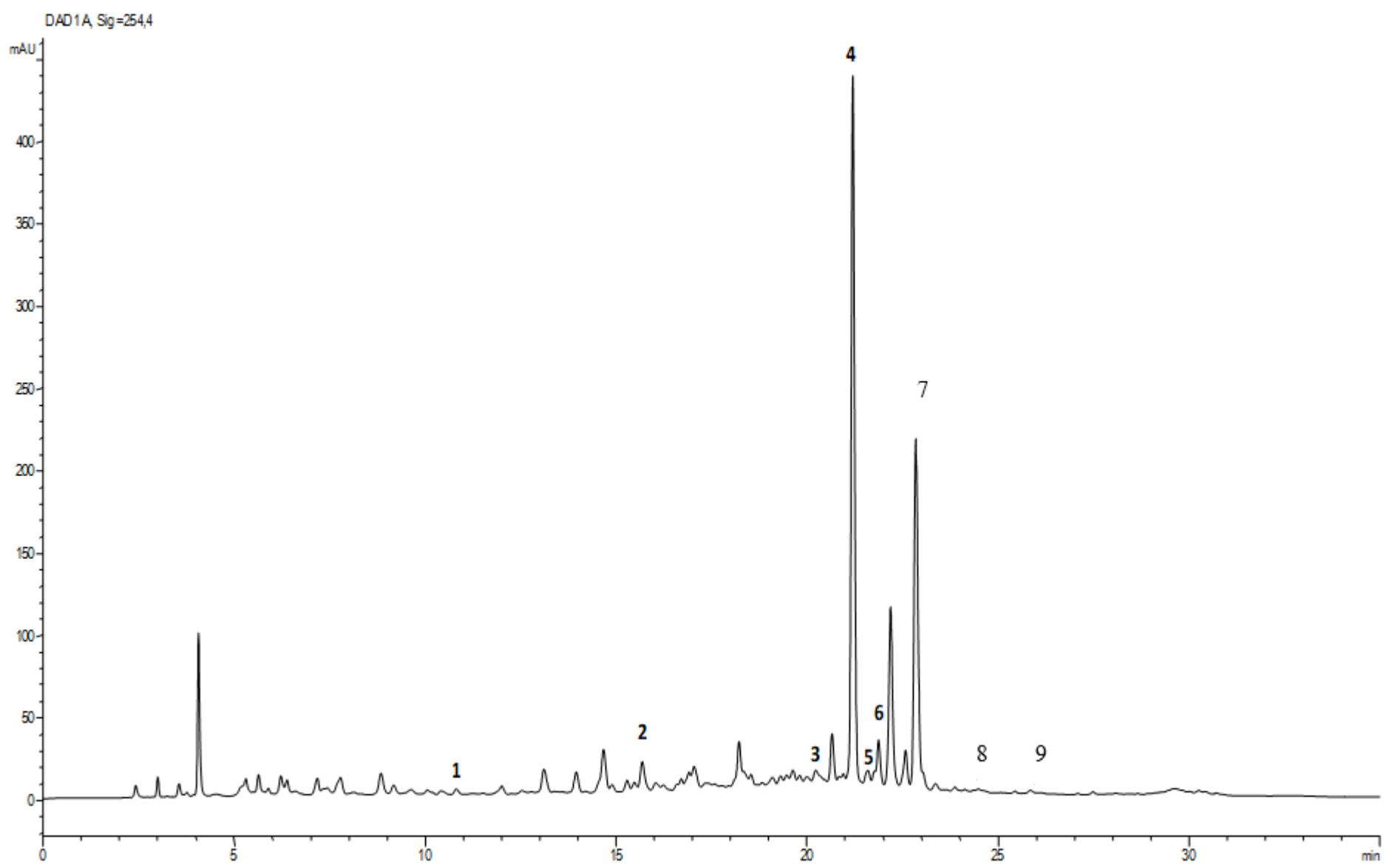

Figure 1

Chromatographic profile of leaf part extract of Nitraria retusa acquired at $245 \mathrm{~nm}$. Peaks were identified based on the retention time of standards. 1: Resorcinol, 2: Chlorogenic acid, 3: Sinapic acid, 4: Luteolin 7-0-glucoside, 5: Isoquercitrin, 6: Hyperoside, 7: Isorhamnetin 3-0- rutinoside, 8: Quercetin, 9: Isorhamnetin. 


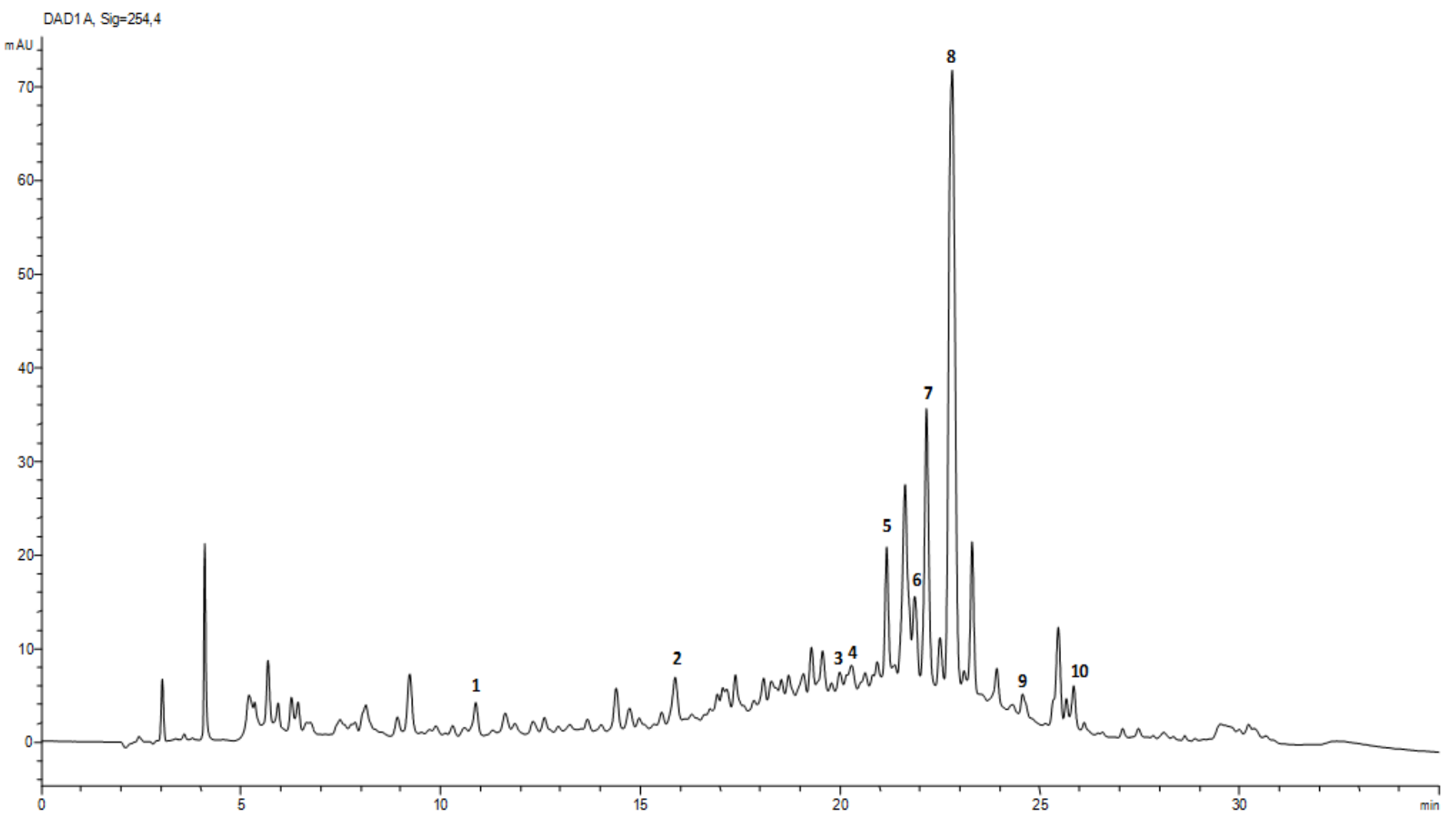

Figure 2

Chromatographic profile of stem part extract of Nitraria retusa acquired at $254 \mathrm{~nm}$. Peaks were identified based on the retention time of standards. 1: Resorcinol, 2: Chlorogenic acid, 3: P-coumaric acid, 4: Sinapic acid, 5: Luteolin 7-0-glucoside, 6: Hyperoside, 7: Isorhamnetin 3-0- rutinoside, 8: Quercetin, 9: Isorhamnetin.

\section{(A)}

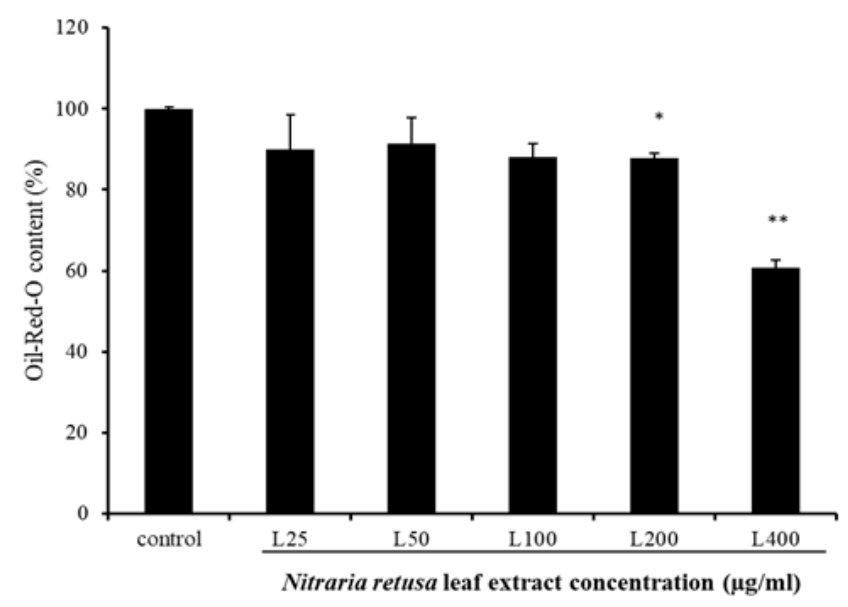

(B)

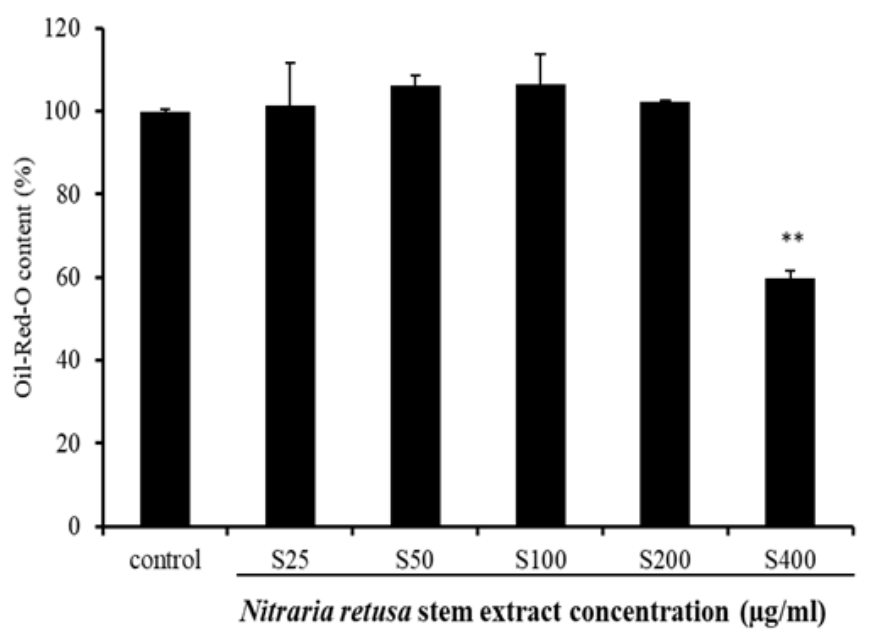

\section{Figure 3}

Effects of Nitraria retusa extracts using different concentrations $(25,50,100,200$ and $400 \mu \mathrm{g} / \mathrm{mL})$ of leaf (A) and Stem (B) on lipid droplet content in 3T3-L1 cells. Lipid droplet accumulation in treated cells was expressed as a percentage of 
control (untreated cells). Fat droplets in adipocytes differentiated for 7 days with or without treatments were stained with oil Red-O dye and relative Oil-Red-O absorbance was measured at $490 \mathrm{~nm}$. Bars represent mean $\pm S D, n=3$, ** $p<0.01$ vs. Control.

(A)

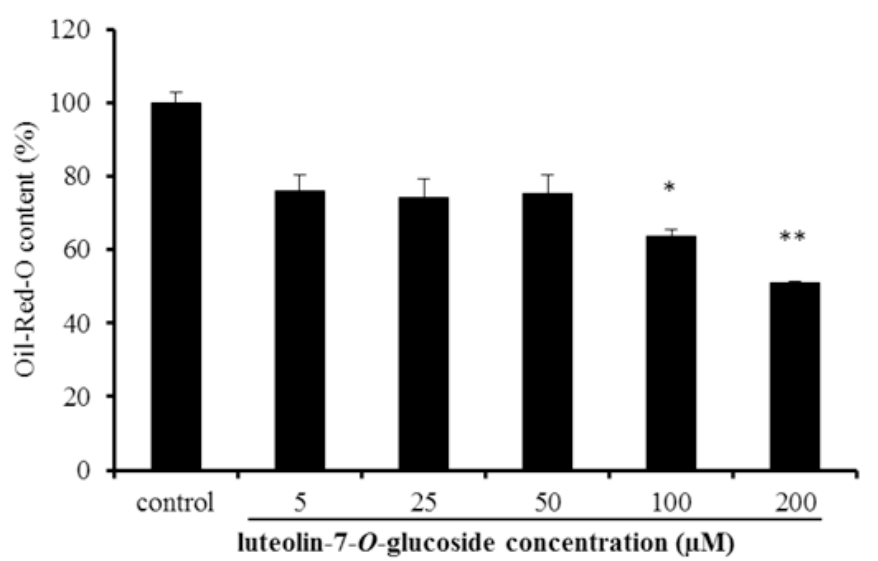

(B)

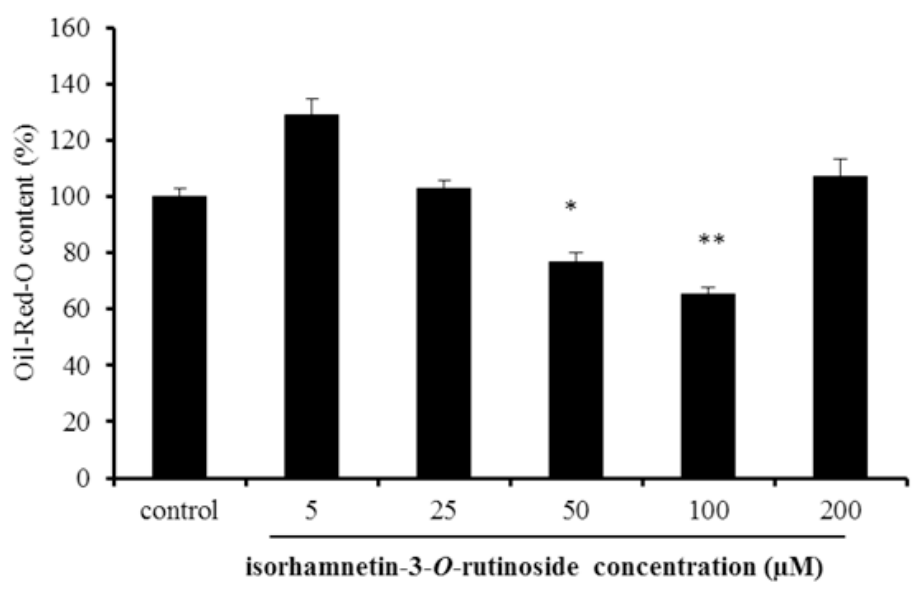

(C)

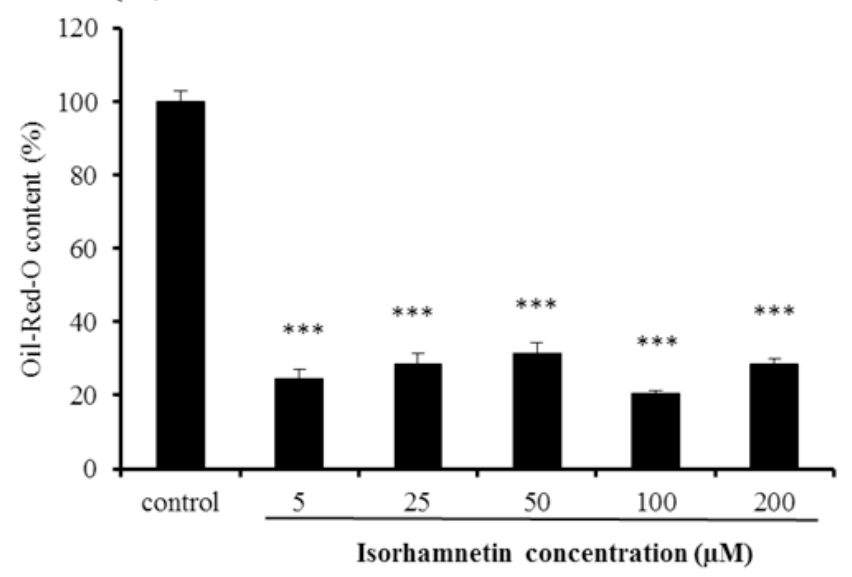

Figure 4

Effects of luteolin-7-0-glucoside (A), isorhamnetin-3-0-rutinoside (B) and isorhamnetin (C) using different concentrations $(5,25,50,100$ and $200 \mu \mathrm{M})$ on lipid droplet content in 3T3-L1 cells. Lipid droplet accumulation in treated cells was expressed as a percentage of control (untreated cells). Fat droplets in adipocytes differentiated for 7 days with or without treatments, were stained with oil Red-0 dye and relative Oil-Red-0 absorbance was measured at 490nm. Bars represent mean $\pm S D, n=3, * p<0.05, * \star p<0.01, * \star * p<0.001$ vs. control. 
(A)

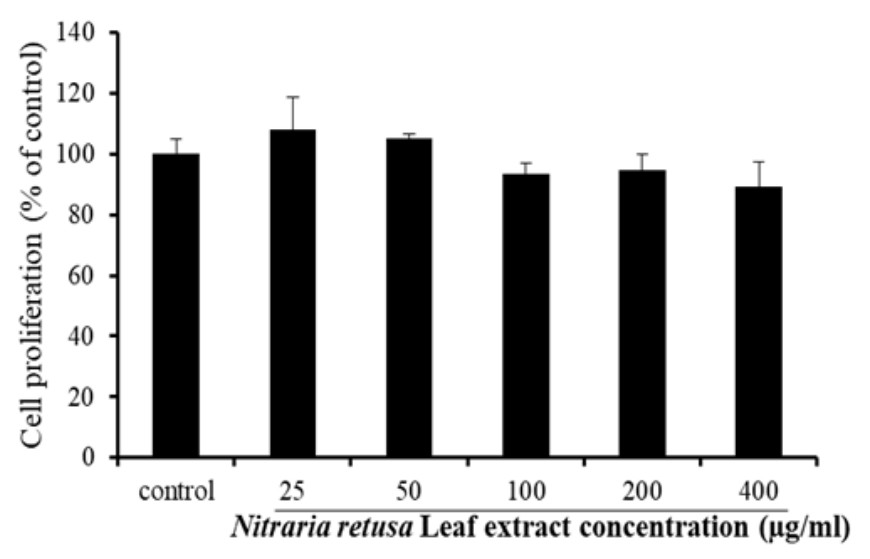

(B)

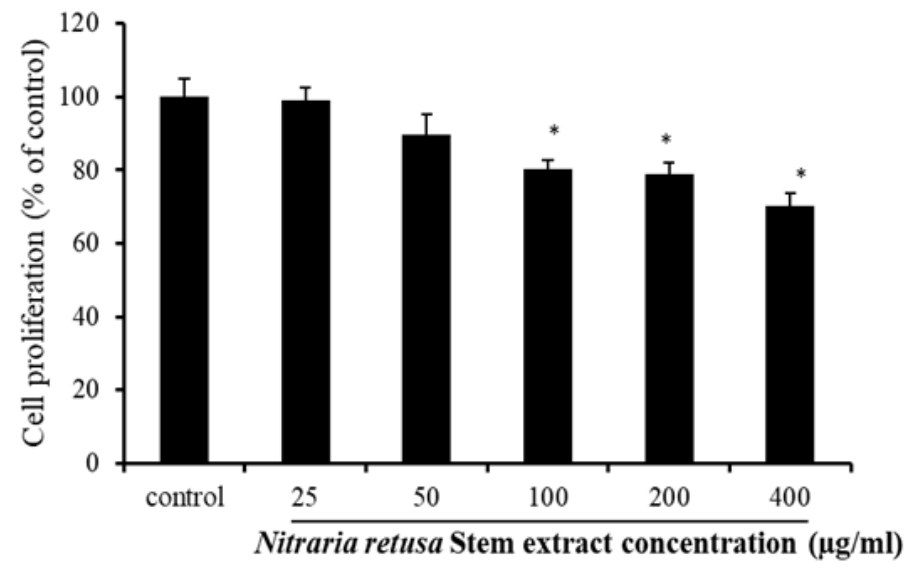

Figure 5

Effects of Nitraria retusa extracts using different concentrations $(25,50,100,200$ and $400 \mu \mathrm{g} / \mathrm{mL})$ of leaf $(A)$ and Stem (B) on the proliferation of 3T3-L1 preadipocytes during differentiation (7 days of treatment). Bars represent mean $\pm S D, n=3$, * $p<0.05$. 
(A)

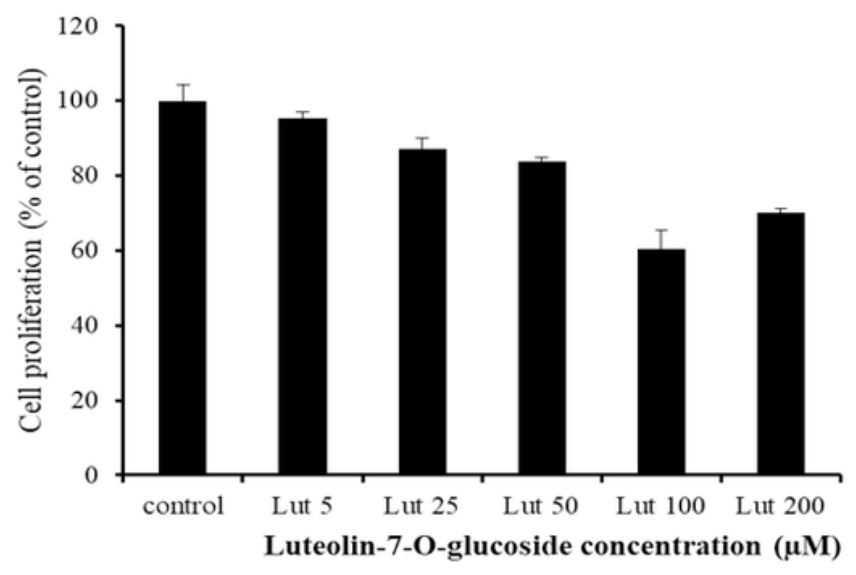

(B)

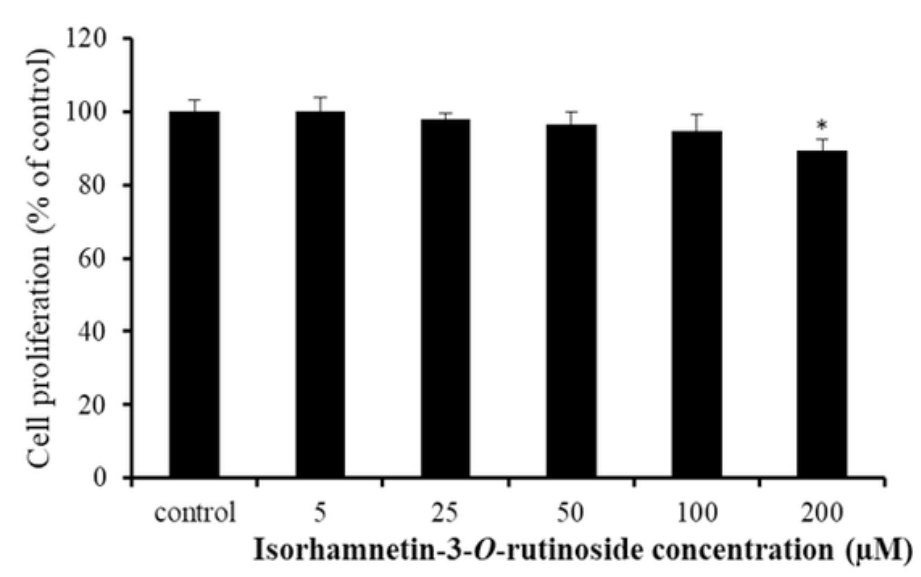

(C)

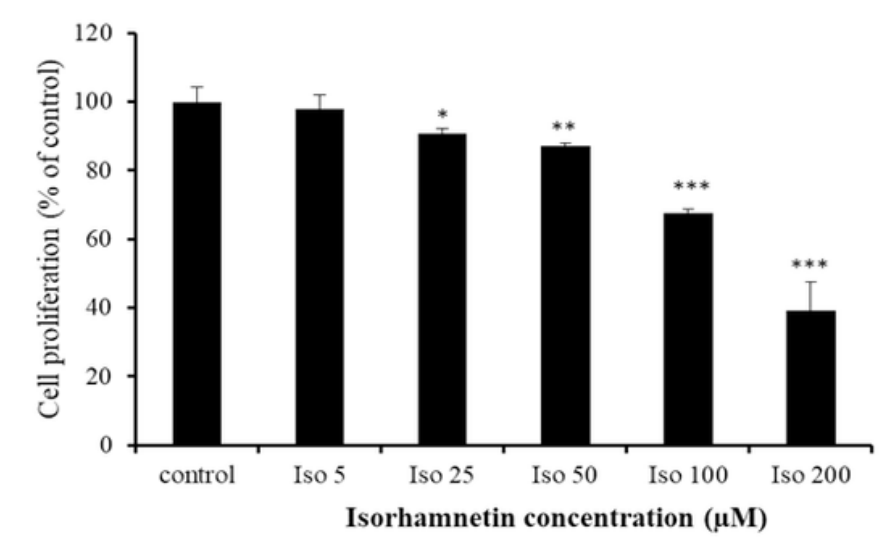

Figure 6

Effects of luteolin-7-O-glucoside (A), isorhamnetin-3-O-rutinoside (B) and isorhamnetin (C) using different concentrations $(5,25,50,100$ and $200 \mu \mathrm{M})$ on 3T3-L1 preadipocytes proliferation during differentiation (7 days of treatment). Bars represent mean $\pm S D, n=3, * p<0.05$, ** $p<0.01$, *** $p<0.001$. 